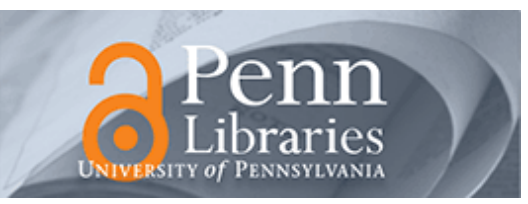

University of Pennsylvania

ScholarlyCommons

December 2004

\title{
Fair Coalitions for Power-Aware Routing in Wireless Networks
}

\author{
Ratul K. Guha \\ University of Pennsylvania \\ Carl A. Gunter \\ University of Pennsylvania \\ Saswati Sarkar \\ University of Pennsylvania, swati@seas.upenn.edu
}

Follow this and additional works at: https://repository.upenn.edu/ese_papers

\section{Recommended Citation}

Ratul K. Guha, Carl A. Gunter, and Saswati Sarkar, "Fair Coalitions for Power-Aware Routing in Wireless Networks", . December 2004.

Copyright 2004 IEEE. Reprinted from Proceedings of the 43rd IEEE Conference on Decision and Control 2004 (CDC 2004), Volume 3, pages 3271-3276.

This material is posted here with permission of the IEEE. Such permission of the IEEE does not in any way imply IEEE endorsement of any of the University of Pennsylvania's products or services. Internal or personal use of this material is permitted. However, permission to reprint/republish this material for advertising or promotional purposes or for creating new collective works for resale or redistribution must be obtained from the IEEE by writing to pubs-permissions@ieee.org. By choosing to view this document, you agree to all provisions of the copyright laws protecting it.

This paper is posted at ScholarlyCommons. https://repository.upenn.edu/ese_papers/145

For more information, please contact repository@pobox.upenn.edu. 


\title{
Fair Coalitions for Power-Aware Routing in Wireless Networks
}

\begin{abstract}
Several power aware routing schemes have been developed under the assumption that nodes are willing to sacrifice their power reserves in the interest of the network as a whole. But, in several applications of practical utility, nodes are organized in groups, and as a result a node is willing to sacrifice in the interest of other nodes in its group but not necessarily for nodes outside its group. Such groups arise naturally as sets of nodes associated with a single owner or task. We consider the premise that groups will share resources with other groups only if each group experiences a reduction in power consuption. When this is the case the groups may form a coalition in which they route each other's packets. We demonstrate that sharing between groups has different properties from sharing between individuals and investigate fair mutually-beneficial sharing between groups. In particular, we propose a pareto-efficient condition for group sharing based on max-min fairness called fair coalition routing. We propose distributed algorithms for computing the fair coalition routing. Using these algorithms we demonstrate that fair coalition routing allows different groups to mutually benefecially share their resources.

\section{Comments}

Copyright 2004 IEEE. Reprinted from Proceedings of the 43rd IEEE Conference on Decision and Control 2004 (CDC 2004), Volume 3, pages 3271-3276.

This material is posted here with permission of the IEEE. Such permission of the IEEE does not in any way imply IEEE endorsement of any of the University of Pennsylvania's products or services. Internal or personal use of this material is permitted. However, permission to reprint/republish this material for advertising or promotional purposes or for creating new collective works for resale or redistribution must be obtained from the IEEE by writing to pubs-permissions@ieee.org. By choosing to view this document, you agree to all provisions of the copyright laws protecting it.
\end{abstract}




\title{
Fair Coalitions for Power-Aware Routing in Wireless Networks
}

\author{
Ratul K. Guha, Carl A. Gunter and Saswati Sarkar \\ University of Pennsylvania \\ \{rguhąaseas, guntera.cis, swatia.ee \}.upenn.edu
}

\begin{abstract}
Several power aware routing schemes have been developed under the assumption that nodes are willing to sacrifice their power reserves in the interest of the network as a whole. But, in several applications of practical utility, nodes are organized in groups, and as a result a node is willing to sacrifice in the interest of other nodes in its group but not necessarily for nodes outside its group. Such groups arise naturally as sets of nodes associated with a single owner or task. We consider the premise that groups will share resources with other groups only if each group experiences a reduction in power consumption. When this is the case the groups may form a coalition in which they route each other's packets. We demonstrate that sharing between groups has different properties from sharing between individuals and investigate fair mutually-beneficial sharing between groups. In particular, we propose a pareto-efficient condition for group sharing based on max-min fairness called fair coalition routing. We propose distributed algorithms for computing the fair coalition routing. Using these algorithms we demonstrate that fair coalition routing allows different groups to mutually benefecially share their resources.
\end{abstract}

\section{INTRODUCTION}

Wireless networks typically consist of nodes that must discharge increasingly complex computing and communication functionalities despite constraints on power, bandwidth, size and memory. Significant progress has been made to improve hardware to address these needs and much is being done to develop software that uses techniques like poweroptimizing algorithms. Comparatively less has been done to exploit sharing amongst nodes as a way to address these challenges. This is unfortunate, since sharing can yield great benefits. A variety of challenges impede progress: (a) determining which resources can be shared, (b) deciding when to share resources, as sharing would evidently involve a cost, (c) deciding with whom to share resources, and (d) determining how to share resources.

Oftentimes, groups of nodes rather than individual nodes are basic entities in the sharing mechanism. The resource expenditure of the group as a whole is more important than that of a single node or the entire network. Groups are often formed on the basis of membership in an organization or a shared task. For example, employees of an organization $A$ may carry computers that belong to $A$. When these devices form an ad hoc network, they may share resources with other devices with the objective of minimizing the total resource consumed by the devices in $A$, rather than that of all devices in the network. Thus, the devices belonging to an organization form a natural group. Wearable computers involved in one distributed computation may form a group. In a sensor network, different groups would consist of

The research was supported in part by NSF EIA00-88028, NSF ANI0106984, ONR N00014-02-1-0715 and NCR-0238340. sensors that measure different attributes such as temperature, pressure etc. In both the above cases, the resource consumed by groups is more important than that consumed by individual nodes as the distributed computation can be performed and the attributes can be measured even when some members fail. The research in this case must investigate issues pertinent to sharing of resources from the perspective of groups.

A group is an intermingled set of nodes having a purpose in common. We do not consider the motivation behind the group formation, but investigate the sharing of resources among different groups. The critical resource we focus on is power. Nodes in wireless networks are powered by battery, and size limitations compel the usage of low lifetime batteries. This calls for judicious consumption of power. Normally, communication consumes higher power than other operations. Nodes share power by routing each others packets, and it is well-known that multihop routing substantially decreases the overall power consumption of the network [25]. We address the research challenges that arise when nodes decide to route each others packets with the sole objective of reducing the power consumption of their groups. We first enumerate these challenges.

The nodes in a group share power by routing each other's packets to common destinations. Groups are said to form coalitions when they route each other's packets. The first challenge is to determine which groups would form coalitions. Presumably, a precondition for forming coalitions among groups is that each group communicates the same amount of information to the chosen destinations while consuming less power after the coalition is formed. Whether or not the precondition is satisfied depends on the routing in the coalition, and the number of possible routes can be an exponential function of the number of nodes in the groups. There need not even exist a routing that reduces the power consumption of each group. Fig.1(a) and (b) show that if each group consists of a single node, then groups do not mutually benefit from the coalition; but this no longer holds if the groups consist of two or more nodes (Fig.1(c)). The challenge then is to answer whether there exists at least one joint routing that makes the coalition mutually beneficial. The next challenge is to decide the joint routing when the coalition is formed. We will show in Section III-C that the routing that minimizes the total power consumption of all groups is not the right choice, as it may increase the power consumption of some groups despite minimizing the power consumption of the network as a whole. The benefit of a group due to the coalition operation is the decrease in its power consumption after it joins the coalition. We need to determine a routing that shares the benefit equitably. A simplistic approach is to insist that the groups each get 


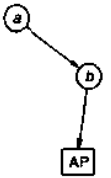

(a)

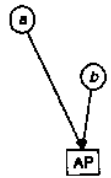

(b) (a)

(a)

AP.
Fig. 1. In (a) and (b), we show two different routings where node $a$ constitutes group A and node $b$ constitutes group B. Both groups need to send traffic to the access point(AP). In (a) the farther node a routes its traffic to $b$ and $b$ sends to AP. So the routing in (a), reduces the power cost of $a$ but increases that for $b$. In (b) each node routes directly to AP and there is no reduction in power costs for both groups.

In (c) nodes $a_{1}$ and $a_{2}$ constitute group $A$ and $b_{1}$ constitutes group $B$. Here $a_{1}$ can send its traffic through $b_{1}$ and $b_{1}$ can in turm send through $a_{2}$. This could result in a decrease in the total power for group $A$ and $B$ as against the case when the groups route to $\mathrm{AP}$ independently.

the same benefit, but this can be wasteful if one group can gain benefit without harming the others. A max-min fair [1] routing uses the following strategy for a pair of groups: determine the greatest minimum benefit to be gained by either of the two groups when sharing and maximize the benefit of the other group so long as the changes do not reduce this minimum. This strategy can be generalized to multiple groups. The challenge now is to compute a maxmin fair power aware coalition routing.

We survey the relevant literature in Section II. We provide a mathematical framework for a coalition of two groups in Section III. This section presents several interesting properties of coalition routings. For example, a max-min fair power aware coalition routing exhibits important characteristics that do not hold for max-min fair allocation of other resources such as bandwidth. We show that the maxmin fair coalition routing is guaranteed to attain the desired minimum benefits for each group should the coalition be feasible. We present a polynomial complexity algorithm for computing the fair coalition routing in Section IV. This algorithm needs solving a linear program at a central processor, which requires the knowledge of the global topology. We present a distributed computing scheme which allows the routing to be computed via simple iterative computations and message exchanges at each participating node in Section V. All proofs can be found in the technical report [9].

\section{RELATED WORK}

The existing research on efficient utilization of power in wireless networks can be classified into the following broad categories. The first maximizes the lifetime of any given node through optimum battery discharge strategy [6], [17]. The second varies the transmission power levels of nodes so as to control the network topology as desired [8], [14], [23]. The third reduces the power consumption by optimizing several parameters at the MAC layer [11], [22]. The last maximizes the lifetime of the network by balancing the power consumption of different nodes [4], [15]. Another prevalent approach is to route in accordance with a power based metric rather than a distance metric [25]. However the common feature of the existing research is that the basic entity is a node. The performance of the network is either quantified in terms of the aggregate performance of the nodes or that of the bottleneck node. However, in our case the basic entity is a group rather than a single node, and the operations are coalitions. The performance objective we consider is fairness and the issues significantly differ on account of the choice of the basic entity. We are concerned about the performance of each group rather than the network as a whole. Relaying and caching strategies have been proposed [21], [18] for node cooperation where a node decides to relay the requests of other nodes. The algorithm in [21] propels the network towards a pareto optimal operating point. Our research is complementary in the sense that we assume that a group of nodes decide to route the packets of other groups based on the interest of the group as a whole. We present an algorithm that obtains a specific pareto optimal objective, the max-min fair operating point.

\section{MATHEMATICAL FRAMEWORK FOR COALITION OF GROUPS}

\section{A. Power Model}

We first present the mathematical model we use for power consumption [7], [24]. Let the transmitted energy per bit be $E_{t}$. Then the received energy at a distance $d$ is $E_{t} d^{-\alpha}$ where $\alpha$ is generally between 2 to 6 . The higher value of the exponent applies for obstructed paths within buildings. We assume that the noise level is the same at all nodes. Let $E_{r}$ be the energy per bit required to maintain a threshold SNR at the receiving end. Then for successful communication $E_{t} d^{-\alpha} \geq E_{r}$. The transmitted power then is of the form $K^{\prime} E_{r} R \vec{d}^{\alpha}$ where $R$ is the bit rate and $K=K^{\prime} E_{r}$ is a constant. We will use $\alpha=4$ which corresponds to the pathloss in closed areas; however all analysis will hold for for any $\alpha \geq 0$.

\section{B. Formulation For a Single Group}

We consider a network in which multiple nodes in a group send traffic to an exit point a(EP). This can be motivated by several commonplace applications. For example, consider a wireless web-cafe, where users send packets to a common access point. In sensor networks measurements must be communicated to exit nodes. In the first case groups can be formed on the basis of membership to different organizations while in the second, groups may be formed on the basis of tasks.

We model the network nodes as a Weighted Directed Graph $\mathbf{G}\langle V, E, \mathbf{a}, W\rangle$ where $V$ is the node set for the group, $E$ is the edge set, $a$ is the exit point and $W$ denotes the edge weights $\in \Re$, $\Re$ denotes the set of real numbers. Every node $v \in V$ has at least one path to node a and outdegree of a is 0 . The node set $V$ and the exit point a are defined through their co-ordinates in the euclidean plane. The distance $d\left(v, v^{\prime}\right)$ is the distance between node $v \in V$ and node $v^{\prime} \in V \cup\{\mathbf{a}\}$. The distance information can be obtained through power measurements and positioning algorithms such as in [2]. Now we define the edge set $E$ and the corresponding weight set $W$. Let $D$ denote the maximum distance that guarantees correct decoding of any communication between two nodes. In other words D ensures an acceptable SNR level at the receiver. A directed edge exists from $v \in V$ to $v^{\prime} \in V \cup\{\mathbf{a}\}$ if $d\left(v, v^{\prime}\right)<D$ and consequently $\left(v, v^{\prime}\right) \in E$ with weight $w\left(v, v^{\prime}\right)=d\left(v, v^{\prime}\right)^{4}$ and $w\left(v, v^{\prime}\right) \in W$. Note that the exit point a has only incoming edges. Origin function $O: V \rightarrow \Re$ defines the 
traffic originating at a node $v \in V$. The graph $\mathbf{G}$ and the origin functions are given.

Let the traffic on an edge $\left(v, v^{\prime}\right)$ be $r\left(v, v^{\prime}\right) \in \Re$. If $\left(v, v^{\prime}\right) \notin E$ then $r\left(v, v^{\prime}\right)=0$. The total outgoing traffic from a node $v$ is then $\sum_{v^{\prime} \in V \cup\{a\}} r\left(v, v^{\prime}\right)$ which is the load on node $v, L(v)$. The sum of the incoming traffic and the originating traffic at a node must equal the exiting traffic. Thus, $\forall v \in V$

$$
\sum_{v^{\prime} \in V \cup\{\mathbf{a}\}} r\left(v, v^{\prime}\right)=O(v)+\sum_{v^{\prime \prime} \in V} r\left(v^{\prime \prime}, v\right)=L(v)
$$

Traffic routing is an $|E|$ dimensional vector $\vec{r}$ whose components satisfy (1). The components of $\vec{r}$ are the traffics on the corresponding edges. Given the routing, the power expenditure of a node $v, N_{\vec{r}}(v)$ is the power spent to transmit load $L(v)$ i.e., $N_{\vec{r}}(v)=K \sum_{v^{\prime} \in V \cup\{\mathbf{a}\}} r\left(v, v^{\prime}\right) d\left(v, v^{\prime}\right)^{4}$ where $K$ is the constant as defined in Section III-A.

The power expenditure of a group $P_{\vec{r}}$ is then the sum of the power expenditure over all nodes of that group i.e., $P_{\vec{r}}=\sum_{\forall v \in V} N_{\vec{r}}(v)$. The group optimal power expenditure $P_{\text {opt }}$ is the minimum value of $P_{\vec{r}}$ over all possible $\vec{r}$. Here $P_{\text {opt }}$ corresponds to routing the traffic over the shortest path from any node $v \in V$ to a in terms of cost metric $W$. The shortest path can be obtained through algorithms like Dijkstra. Let $v^{\prime}$ be the next hop node to $v$ as obtained from the shortest path algorithm. If $N_{\text {opt }}(v)$ is the power spent by a node $v$ under optimal routing, then

$N_{o p t}(v)=K \times L(v) \times d\left(v, v^{\prime}\right)^{4}$ and $P_{o p t}=\sum_{\forall v \in V} N_{o p t}(v)$.

\section{Coalition of Groups}

We have described the terminology and the equations for a group of nodes. Now consider two groups of nodes A and $\mathrm{B}$. Let their node sets be $V^{a}$ and $V^{b}$ respectively and optimal power expenditures before forming a coalition be $P_{o p t}$ and $P_{o p t}^{b}$.

Next we consider a combined network with groups $A$ and $B$ jointly routing to the exit point. The vertex set $V$ for the combined network then is $V^{a} \cup V^{b}$. The edge set $E^{\text {joint }}$ can be found from $V$ as follows. A directed edge exists from $v \in V^{a} \cup V^{b}$ to $v^{\prime} \in V^{a} \cup V^{b} \cup\{\mathbf{a}\}$ if $d\left(v, v^{\prime}\right)<D$ and consequently $\left(v, v^{\prime}\right) \in E^{\text {joint }}$ with weight $w\left(v, v^{\prime}\right)=$ $d\left(v, v^{\prime}\right)^{4}$. The origin functions for all the nodes remain the same. A coalition routing in this network is a vector whose components satisfy (1). Note that $r\left(v, v^{\prime}\right)=0$ if $\left(v, v^{\prime}\right) \notin$ $E^{j o i n t}$. For an arbitrary coalition routing $\vec{r}$, evaluate the power expenditure for each node. Let $J_{\vec{r}}^{a}$ and $J_{\vec{r}}^{b}$ be the total power expenditure for nodes in groups $\mathrm{A}$ and $\mathrm{B}$ respectively, under routing $\vec{r}$.

$$
J_{\vec{r}}^{a}=\sum_{\forall v \in V^{a}} N_{\vec{r}}(v) \text { and } J_{\vec{r}}^{b}=\sum_{\forall v \in V^{b}} N_{\vec{r}}(v) .
$$

Definition 1: Group benefit under coalition routing $\vec{r}$ is the difference between the power spent by the group under individual optimal routing before merging, and the power spent by the group for coalition routing $\vec{r}$.

The group benefits under routing $\vec{r}$ form the benefit vector $\vec{B}_{\vec{r}}$. Hence the benefit vector is $\vec{B}_{\vec{r}} \equiv\left(B_{\vec{r}}^{a}, B_{\vec{r}}^{b}\right)$ with components $B_{\vec{r}}^{a}=P_{o p t}^{a}-J_{\vec{r}}^{a}$ and $B_{\vec{r}}^{b}=P_{o p t}^{b}-J_{\vec{r}}^{b}$.

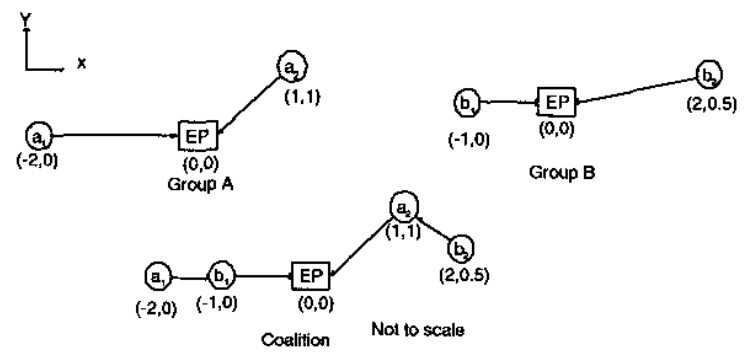

Fig. 2. Groups $\mathrm{A}\left(a_{1}, a_{2}\right)$ and $\mathrm{B}\left(b_{1}, b_{2}\right)$ route to the exit point EP. Each node sends $1 \mathrm{Mbps}$

The idea behind combining two groups is to reduce the total power each group was spending initially. Depending on the system, group coalition may introduce some additional operational cost and groups would want to benefit over and above this cost

Definition 2: A coalition is useful with a routing $\vec{r}$ if $\min \left(B_{\vec{r}}^{a}, B_{\vec{r}}^{b}\right) \geq t$. A coalition is useful if there exists a routing $\vec{r}$ such that the coalition is useful with routing $\vec{r}$. We would present an algorithm to compute such a routing $\vec{r}$ if one exists. The choice of the threshold $t$, would depend on group policies and the overhead for the coalition.

Definition 3: A Minimal coalition routing is a joint routing that results in the optimal or the minimal total power expenditure for groups A and B combined.

Next we illustrate the combination of two groups with an example. Consider Fig. 2 in which groups $A$ and $B$ route to the exit point. Each node generates traffic at the rate of 1Mbps. Optimal power expenditure for group $\mathrm{A}$ is $2^{4}+$ $\sqrt{2}^{4}=20$ and for group $B$ is $1^{4}+\sqrt{4.25}^{4} \approx 19$. For the minimal power coalition routing shown, power expenditure for $A$ is $1^{4}+2(\sqrt{2})^{4}=9$ and for $B$ is $2(1)^{4}+\sqrt{1.25}^{4} \approx 3.6$. Benefit for group $\mathrm{A}$ is $20-9=11$ and for $\mathrm{B}$ is $19-3.6=$ 15.4 and both the components are positive. Consider now that node $b_{2}$ has a higher load to send, e.g., $5 \mathrm{Mbps}$. This will be relayed through $a_{2}$ in the coalition routing of Fig.2. Node $a_{2}$ will have a high power consumption(24) and the benefit of group $A$ will be negative(-5). This illustrates that the minimal coalition routing may not benefit each group.

Definition 4: A feasible benefit vector is one that results from a coalition routing $\vec{r}$ that satisfies (1). The set of all feasible benefit vectors is the feasible benefit region.

\section{Properties of the Feasible Benefit Region}

For the minimal coalition routing, we can find the power expenditure for each node, i.e. $N_{o p t}(v)$ for each $v \in\left(V^{a} \cup\right.$ $V^{b}$ ). Further let $J_{o p t}^{a}$ and $J_{o p t}^{b}$ be the power spent by nodes of group $A$ and $B$ respectively under the minimal coalition routing.

$$
J_{o p t}^{a}=\sum_{\forall v \in V^{a}} N_{o p t}(v) \text { and } J_{o p t}^{b}=\sum_{\forall v \in V^{b}} N_{o p t}(v) .
$$

Note again that the subscript 'opt' to $J$ refers to minimal coalition routing for nodes of group $\mathrm{A}$ and $\mathrm{B}$ combined. The benefit vector $\vec{L}$ corresponding to the minimal coalition routing is then $\left(L_{\text {opt }}^{a}, L_{\text {opt }}^{b}\right)$ where $L_{\text {opt }}^{a}=P_{\text {opt }}^{a}-$ $J_{o p t}^{a}$ and $L_{o p t}^{b}=P_{o p t}^{b}-J_{o p t}^{b}$. The vector $\vec{L}$ is plotted in Fig. 3 for different random placements of nodes. Each 


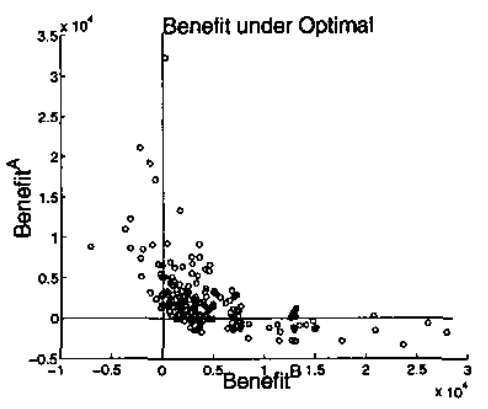

Fig. 3. 'Benefit vectors" under 'minimal coalition'

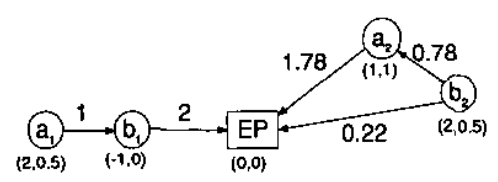

Fig. 4. Fair coalition routing when each node sends $1 \mathrm{Mbps}$. The numbers next to the links are the rates.

group has 20 nodes spread over a a square of side $100 \mathrm{~m}$. If the benefit vector is in the first quadrant (both coordinates are positive), then the groups mutually benefit from being merged, otherwise one of the groups is a loser. Most pairs of groups benefit from a minimal coalition, but there are many instances in which only one group benefits. Even when a pair of groups mutually benefits, there is often some disproportion in the extent of benefit, with one group getting somewhat more than the other.

Theorem 1: The set of feasible benefit vectors is convex and closed.

\section{E. Max-min Fair Benefit Vector}

Definition 5: A feasible benefit vector $B_{\vec{r}}$ is max-min fair if $\forall i, B_{\vec{r}}^{i}$ cannot be increased while maintaining feasibility without decreasing $B_{\vec{r}}^{j}$ for some group $j$, for which $B_{\vec{r}}^{j} \leq B_{\vec{r}}^{i}$.

Corollary 1: The max-min fair benefit vector exists and is unique.

The corollary follows as a consequence of Theorem 1 and results from [19].

Definition 6: A Fair Coalition routing is a joint routing that results in a max-min fair benefit vector.

In Fig.2 the max-min fair benefit vector is $(11.9,11.9)$. This is achieved when node $b_{2}$ sends $0.78 \mathrm{Mbps}$ to $a_{2}$ and $0.22 \mathrm{Mbps}$ directly to AP like in Fig.4.

Proposition 1: Let $\vec{r}$ be a fair coalition routing. Then $\min \left(B_{\vec{r}}^{a}, B_{\vec{F}}^{b}\right) \geq 0$.

Thus a coalition does not increase the power consumption of any group if fair coalition routing is used.

Theorem 2: A coalition will be useful if and only if it is useful with a fair coalition routing $\vec{r}$.

Theorem 2 presents a necessary and a sufficient condition for deciding whether the coalition would be useful.

Theorem 3: For two groups the max-min fair benefit vector has equal components.

Theorem 3 will be used in developing an efficient algorithm for computing a fair coalition routing for two groups. Note that for other resource allocation problems e.g. bandwidth allocation, the max-min fair vector need not

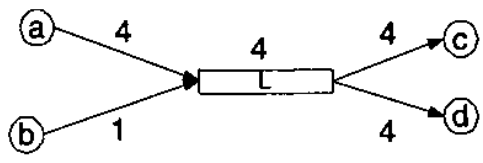

Fig. 5. Consider two sessions (a,c) and (b,d). The numbers next to the links are the link bandwidths. The max-min fair bandwidth for session $(\mathrm{a}, \mathrm{c})$ and $(\mathrm{b}, \mathrm{d})$ are 3 and 1 respectively.

have equal components even for two contenders (Fig.5) [5]. Theorem 3 does not hold for fair power allocation although it holds for fair benefit allocation [9].

\section{FAIR COALITION ALGORITHM(FC)}

\section{A. Description}

We show that the fair coalition routing and the associated benefit vector can be computed by solving the following linear program.

FC: Maximize $Z$ :

Subject to:

$$
\begin{aligned}
& Z \leq B_{\overrightarrow{\vec{r}}}^{\mathbf{a}}, \\
& Z \leq B_{\overrightarrow{\mathbf{r}},}^{\mathbf{b}}, \\
& \sum_{v^{\prime} \in V^{a} \cup V^{b} \cup\{\mathbf{a}\}} r\left(v, v^{\prime}\right)=O(v)+\sum_{v^{\prime \prime} \in V^{a} \cup V^{b}} r\left(v^{\prime \prime}, v\right) \forall v, v^{\prime} \in V^{a} \cup V^{b} .
\end{aligned}
$$

where

$$
\begin{gathered}
B_{\vec{r}}^{a}=P_{o p t}^{a}-J_{\vec{r}}^{a} \text { and } B_{\vec{r}}^{b}=P_{o p t}^{b}-J_{\vec{r}^{4}}^{b} \\
J_{\vec{r}}^{a}=K \sum_{\forall v \in V^{a}} \sum_{v^{\prime} \in V^{a} \cup V^{b} \cup\{\mathbf{a}\}} r\left(v, v^{\prime}\right) d\left(v, v^{\prime}\right)^{4} . \\
J_{\vec{r}^{b}}^{b}=K \sum_{\forall v \in V^{b} v^{\prime} \in V^{a} \cup V^{b} \cup\{\mathbf{a}\}} r\left(v, v^{\prime}\right) d\left(v, v^{\prime}\right)^{4} .
\end{gathered}
$$

Let $Z^{*}$ be the objective function value obtained from $\mathbf{F C}$.

Theorem 4: The routing $\vec{r}$ obtained as a solution of $\mathbf{F C}$ is a fair coalition routing.

The linear program involves $|V|+2$ constraints and $|E|+1$ variables. Hence the max-min fair benefit vector and the fair coalition routing are polynomial complexity computable [13].

\section{B. Simulation Results}

We investigate the efficacy of fair coalition routing through simulations using MATLAB. Specifically we will be interested in comparing the performance of fair coalition routing with the minimal coalition routing. The value of $K$ depends on the choice of the wireless interface, and its effect is to scale our measurements. Thus without loss of generality we consider $K=1$. We will later mention details for a specific interface.

We consider a square of side $100 \mathrm{~m}$. The exit point is at the center of the square. We consider a fully connected network in which each node can transmit directly to every other node. We consider a coalition of two groups in 100 random topologies. In Fig.6(a), we investigate the case when both the combining groups have equal number of nodes. Nodes of the combining groups are uniformly distributed over the square area. The max-min fair benefit vector will always have equal components in this case. We average over the maximum component of the optimal and the minimum of the optimal over all the topologies. As expected the 


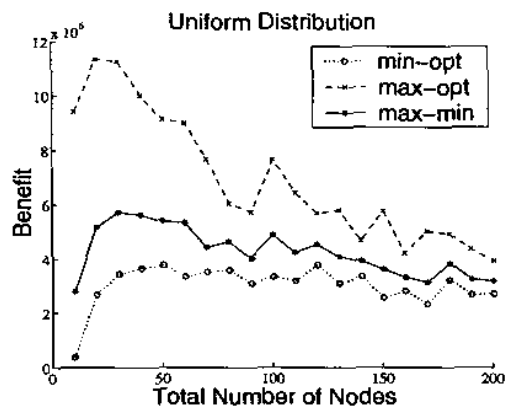

(a) Equal group sizes

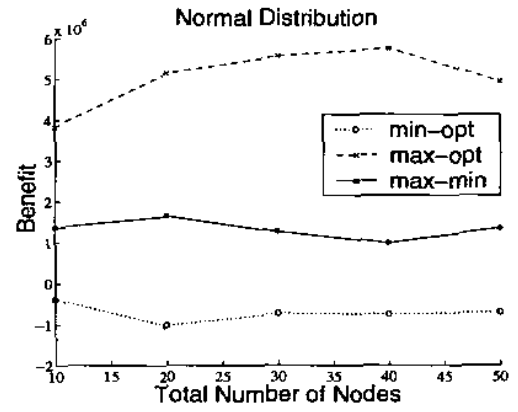

(d) Clustered Topology

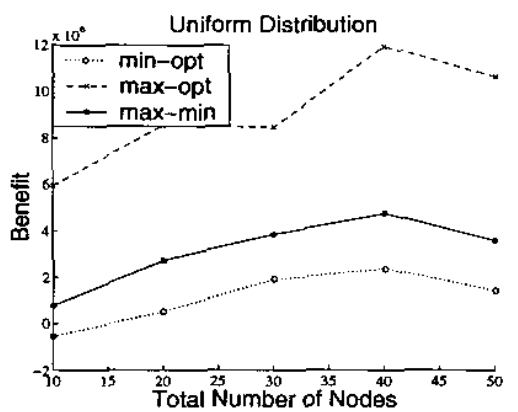

(b) Skewed Group Sizes

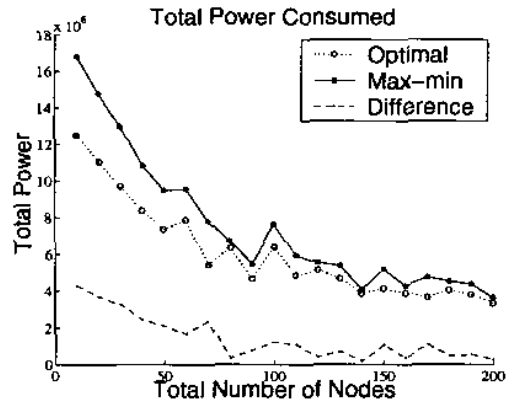

(e) Fairness Overhead

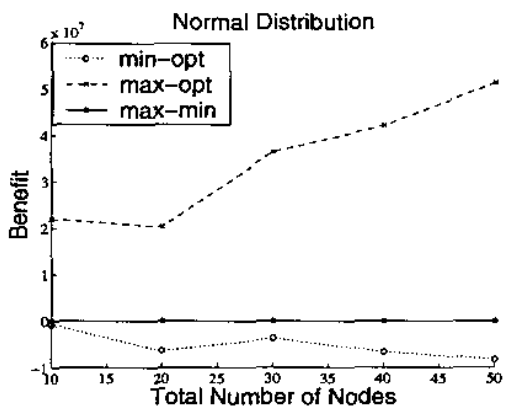

(c) Clustered Groups

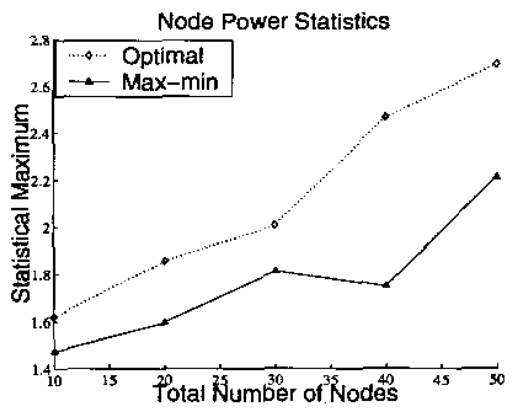

(f) Effect on Lifetime

Fig. 6. Results for two groups spread over a square of side $100 \mathrm{~m}$. Max-opt denotes the average over the maximum component of the optimal and min-opt denotes the average over the minimum component of the optimal. The average is over 100 random topologies

max-min group benefit lies between the maximum and the minimum components of the optimal. The benefit obtained for group merger is less pronounced for sizes more than 50-60 nodes. Therefore we will consider networks of size up to 50 nodes subsequently. Fig.6(b) shows the results for unequal group sizes. One group is four times larger than the other. The smaller group has a lesser benefit under the optimal in this case. The remaining trends are the same as in the previous case. Fig.6(c) studies the effect of clustered topologies on the benefit values. Nodes of each group are normally distributed around a randomly chosen center. In each case the group with the center closer to the exit point has negative benefit. This group will suffer under coalition routing but in the max-min fair case it has zero benefit, and hence it does not lose. In Fig.6(d) we consider a similar clustered topology where the clusters include nodes from both groups. The trends are similar to Fig.6(c), but both groups obtain positive benefits under fair coalition. Fig.6(e) plots the total power spent under the minimal coalition routing, fair coalition routing and their difference. This difference can be looked upon as the cost for providing fairness. The average cost is modest $(18 \%)$ considering the benefit $(46 \%)^{1}$ obtained and the fairness achieved.

Oftentimes lifetime of a network is determined by the node that spends the maximum power [3], [4], [25]. Thus

\footnotetext{
${ }^{1}$ The cost $\%$ is obtained from Fig.6(e). The benefit $\%$ is with respect to the total power consumed prior to the coalition and is obtained from Fig.6(a) and Fig.6(e).
}

in Fig.6(f) we plot the quantity $\left(\bar{X}+\sigma_{x}\right) / \bar{X}$ where $\bar{X}$ is the mean power over all nodes and $\sigma_{x}$ is the standard deviation. Note that this quantity is a measure of the statistical maximum of the power spent by any node. Fair coalition routing has a lower value of this quantity as compared to the minimal.

For the Lucent $802.11 \mathrm{~b}$ Orinoco card, a rate of $1 \mathrm{Mbps}$ in closed environment corresponds to $15 \mathrm{dBm}$ of output power [16]. The constant $K$ is then roughly $5.5 \times 10^{-6} \mathrm{~W} / \mathrm{Mbit} *$ $m^{4}$. This translates to a benefit of 30 Watts for a group with 10 nodes for the uniform case with equal group sizes. It is also notable that the CPU time to compute FC, for any considered topology was not more than 0.5 secs on a $700 \mathrm{Mhz} / 256 \mathrm{MB}$ RAM laptop using a simplex algorithm implementation [10].

\section{DISTRIBUTED IMPLEMENTATION}

The algorithm in Section IV-A for computing the fair coalition routing requires a centralized computation at the exit point. Though the simplest solution, it will not be computationally tractable when the exit points have capability similar to the nodes themselves. Consider for example a sensor network where a group of sensors communicate their measurements to a common node which in turn transmits to say a satellite. Here we would not want to overwhelm the relay node with the linear programming computation. Instead it would be beneficial to have a distributed implementation where every node performs some simple iterative 
computation and the values converge to the max-min fair solution. The iterative approach has been motivated by recently proposed solutions for optimization problems in other resource allocation settings [12], [20].

\section{A. Iterative Algorithm}

Now we present an iterative approach to compute fair coalition routing for two groups. Let $Z_{n}$ and $\vec{r}_{n}$ denote the corresponding quantities in iteration $n$, where $Z_{0}$ and $\overrightarrow{r_{0}}$ can be arbitrarily chosen. The initial choices need not satisfy any of the constraints. Thus each node can select the initial values of the loads for each of its outgoing edges without any co-ordination with the other nodes. Similarly $Z_{0}$ is selected at the exit point. Now we define some indicators. The benefit indicator of a group is 1 if $Z_{n}$ is more than the group benefit.

$$
\begin{aligned}
& \epsilon_{n}^{a}=\left\{\begin{array}{lll}
0 & \text { if } & Z_{n}+J_{r_{n}}^{a} \leq P_{o p t}^{a} \\
1 & \text { if } & Z_{n}+J_{r_{n}}^{a}>P_{o p t}^{a}
\end{array} \epsilon_{n}^{b}=\left\{\begin{array}{lll}
0 & \text { if } & Z_{n}+J_{r_{n}}^{b} \leq P_{o p t}^{b} \\
1 & \text { if } & Z_{n}+J_{r_{n}}^{b}>P_{o p t}^{b}
\end{array}\right.\right.
\end{aligned}
$$

Node congestion $c_{n}^{v}$ is the difference between the outgoing and the sum of the originating and incoming traffic at node

$c_{n}^{v}=\sum_{v^{\prime} \in V^{a} \cup V^{b} \cup\{\mathbf{a}\}} r_{n}\left(v, v^{\prime}\right)-\left(O(v)+\sum_{v^{\prime \prime} \in V^{a} \cup V^{b}} r_{n}\left(v^{\prime \prime}, v\right)\right)$.

Node congestion indicator for node $v \in V^{a} \cup V^{b}$ is

$$
s_{n}^{v}=\left\{\begin{array}{rll}
0 & \text { if } & c_{n}^{v}=0 \\
1 & \text { if } & c_{n 2}^{v}>0 \\
-1 & \text { if } & c_{n}^{v}<0
\end{array}\right.
$$

Node $v$ is considered balanced, lightly loaded or heavily loaded as $s_{n}^{v}$ is 0,1 and -1 respectively. For the exit point, $s_{n}^{\mathbf{a}}=0$. We present an iterative approach using the above indicators. Note that $s_{n}^{v}$ can be updated at node $v$ using the incoming rates in the previous iteration. Now, update of $\epsilon_{n}^{a}$ and $\epsilon_{r_{b}}^{b}$ require a knowledge of the total power being spent by the nodes of a group. This information can be acquired in a distributed manner as shown in [9].

Let $\left\{\delta_{n}\right\}$ be the step-sizes that satisfy $\lim _{n \rightarrow \infty} \delta_{n}=0$ and $\sum_{n=1}^{\infty} \delta_{n}=\infty$. For example $\delta_{n}=1 / n$ satisfies the conditions. Each node updates its outgoing traffic as follows. []$_{+}$denotes the projection on $[0, \infty)$.

$r_{n+1}\left(v, v^{\prime}\right)=\left[r_{n}\left(v, v^{\prime}\right)-\gamma \delta_{n}\left(s_{n}^{v}-s_{n}^{v^{\prime}}+d\left(v, v^{\prime}\right)^{4} \epsilon_{n}^{a}\right)\right]_{+}$ if $v \in V^{a}$.

$r_{n+1}\left(v, v^{\prime}\right)=\left[r_{n}\left(v, v^{\prime}\right)-\gamma \delta_{n}\left(s_{n}^{v}-s_{n}^{v^{\prime}}+d\left(v, v^{\prime}\right)^{4} \epsilon_{n}^{b}\right)\right]_{+}$ if $v \in V^{b}$.

The exit point updates $Z$ as follows.

$$
Z_{n+1}=\left[Z_{n}+\delta_{n}\left(1-\gamma\left(\epsilon_{n}^{a}+\epsilon_{n}^{b}\right)\right)\right]_{+} \text {. }
$$

Theorem 5: For all $\gamma>1$ the iterative procedure stated above will converge to the max-min fair benefit vector and fair coalition routing, irrespective of the initial choice of the iterates.

\section{Conclusions}

We have studied the problem of forming coalitions between groups of nodes with the intent of saving power. We found that an application of max-min fair techniques to this problem yields an efficient and balanced approach which we call fair coalition routing. We developed theory and algorithms for fair coalition routing. We have carried out a range of simulations that demonstrate that fair coalition routing is practical and beneficial in common cases. We generalize the framework and the computation algorithms for a coalition among multiple groups in [9]. The coalition routing algorithms presented in this paper provide foundations for developing operational protocols. Design of such protocols would require deployment of mechanisms to enforce group routings e.g., security checks. We discuss some of these issues in [9].

Acknowledgements. We appreciated comments from Ron Brachman and Bob Hickok.

\section{REFERENCES}

[1] Dimitri Bertsekas and Robert Gallager. Data Networks. Prentice Hail, 1992.

[2] S. Capkun, M. Hamdi, and J. P. Hubaux. Gps-free positioning in mobile ad hoc networks. Prac. Hawaii Int. Conf. on System Sciences, 2001.

[3] J. Chang and L. Tassiulas. Routing for maximum system lifetime in wireless ad hoc networks. In Proc. 37th Annual Allerton Conference on Communication, Control and Computing, 1999.

[4] J. Chang and L. Tassiulas. Energy conserving routing in wireless ad-hoc networks. Proc. of the IEEE INFOCOM, Tel Aviv, 2000.

[5] $S$. Chen and $K$. Nahrstedt. Maxmin fair routing in connectionoriented networks. Proc. of Euro-Parallel and Distributed Systems Conference, 1998.

[6] C. F. Chiasserini and R. R, Rao. Energy efficient battery management. Proc. of the IEEE INFOCOM, Tel Aviv, 2000.

[7] S. Doshi, S. Bhandare, and T. X. Brown. An on-demand minimum energy routing protocol for a wireless ad hoc network. Mobile Computing and Communications Review, 6(3), July 2002.

[8] T. A. ElBatt, S. V. Krishnamurthy, D. Connors, and S. Dao. Power management for throughput enhancement in wireless ad-hoc networks. IEEE International Conference on Communications, 2000.

[9] R. K. Guha, C. A. Gunter, and S. Sarkar. Fair coalitions for poweraware routing in wireless networks. Technical report, University of Pennsylvania, http://einstein. seas.upenn.edu/mnlab/publications.html, 2004.

[10] F. S. Hillier and G. J. Lieberman. Introduction to Mathematical Programming. McGraw-Hill, Inc., 1995.

[11] E. Jung and $\mathbf{N}$. $H$. Vaidya. An energy efficient mac protocol for wireless lans. Proc. of the IEEE INFOCOM, NY, 2002 .

[12] K. Kar, S. Sarkar, and L. Tassiulas. A simple rate control algorithm for maximizing total user utility. Proc. of the IEEE INFOCOM Anchorage, 2001.

[13] N. Karmarkar. A new polynomial-lime algorithm for linear programming. Combinatorica, 4:373-395, 1984.

[14] V. Kawadia and P. R. Kumar. Power control and clustering in ad hoc networks. Proc. of the IEEE INFOCOM, San Francisco, 2003.

[15] Q. Li, J, Aslam, and D. Rus, Online power-aware routing in wireless ad-hoc networks. Proc. of Mobicom, 2001.

[16] Lucent Technologies. Orinoco PC Card Guide, August 2000.

[17] S. Sarkar M. Adamou. A framework for optimal battery management for wireless nodes. Proc. of the IEEE INFOCOM, NY, 2002.

[18] P. Nuggehalli, V. Srinivasan, and C. Fabiana. Energy-efficient caching strategies in ad hoc wireless networks. Proc. of ACM Mobihoc, 2003

[19] Saswati Sarkar and Kumar N. Sivarajan. Fairness in cellular mobile networks. IEEE Transactions of Information theory, 48(8):24122426, Aug 2002.

[20] V. Srinivasan, C. Chiasserini, P. Nuggehalli, and R. R. Rao. Optimal rate allocation and traffic splits for energy efficient routing in ad hoc networks. Proc. of the IEEE INFOCOM, NY, 2002

[21] V. Srinivasan, P. Nuggehalli, C. Chiasserini, and R. R. Rao. Cooperation in wireless ad hoc networks. Proc. of the IEEE INFOCOM, San Francisco, 2003.

[22] Y. Tseng, C. Hsu, and T. Hsieh. Power-saving protocols for iece 802.1 1-based multi-hop ad hoc networks. Proc. of the IEEE INFOCOM, $N Y, 2002$.

[23] Roger Wattenhofer, Li Li, Paramvir Bahl, and Yi-Min Wang. Distributed topology control for power efficient operation in multihop wireless ad hoc networks. Proc. of the IEEE INFOCOM, Anchorage, 2001.

[24] J. E. Wieselthier, G. D. Nguyen, and A. Ephremides. Resourcelimited energy-efficient wireless multicast of session traffic. Proc. of the 34th Hawaii International Conference on System Sciences, 2001.

[25] M. Woo, S. Singh, and C. S. Raghavendra. Power aware routing in mobile ad hoc networks. Proc. of ACM Mobicom 98, 1998. 